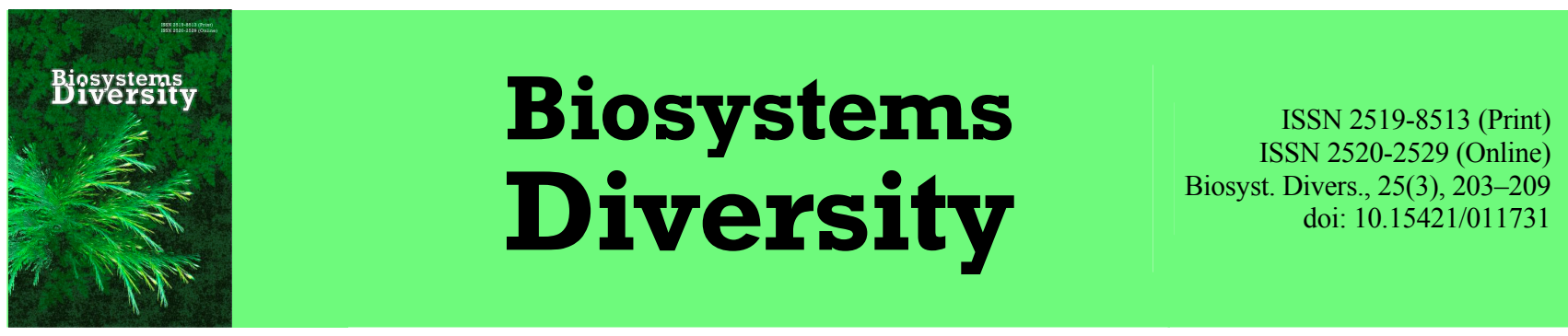

\title{
Model of influence of landscape vegetation on mass transfer processes
}

\author{
L. D. Romanchuck, T. P. Fedonyuk, R. G. Fedonyuk \\ Zhytomyr National Agroecologycal University, Zhytomyr, Ukraine
}

Article info

Received 12.04.2017

Received in revised form 07.05.2017

Accepted 10.05.2017

Zhytomyr National

Agroecologycal University, Staryj

Bul'var, 7, Zhytomyr

10002, Ukraine.

Tel.: +38-041-222-75-19.

E-mail:

tanyavasiluk2015@gmail.com

\author{
Romanchuck, L. D., Fedonyuk, T. P., \& Fedonyuk, R. G. (2017). Model of influence of landscape vegetation on \\ mass transfer processes. Biosystems Diversity, 25(3), 203-209. doi:10.15421/011731
}

The problem of mass transfer of landscape is an important and urgent problem which has actively been elaborated during the last several decades. In particular, the problem of interaction between two-phase wind flow and landscape vegetation is a key to understanding the evolution of landscape morphology, pollution distribution and soil erosion. In this context the mathematical modeling of mass transfer processes within complex environments is an advanced tool necessary for better understanding of environmental processes. In this article, a mathematical model describing the processes of mass transfer on an inhomogeneous surface in a porous environment has been developed and theoretically investigated. The mechanical impact of boundary surfaces and porous environment structure on a mass transfer process has been considered and included into the model. The mass source function adapted to the specific inhomogeneous domain has been developed and investigated. In this paper we develop a formal framework to reflect correctly the problem of landscape mass transfer within the vegetation by incorporating it into a formal system with a reduced number of dependent variables and simplified boundary conditions. We develop a mathematical model of mass transfer process realized on an inhomogeneous boundary surface. The mechanical impact of boundary surface on the mass transfer process has been considered and taken into account. The mechanical impact of porous environment structure on mass transfer process has also been considered and taken into account. The substance source function has been developed here.

Keywords: pollution; soil erosion; porous environment structure; two-phase wind

\section{Introduction}

The problem of study of horizontal landscape mass transfer processes in the context environmental safety of particular territories has grown quite urgent recently. On the one hand, this is due to the intensive cultivation of the environment, on the other hand, it is due to the transition to a new qualitative and quantitative level of the state of the environment, caused by the accumulation of gradual changes over many decades.

According to data from the literature, dust storms are an almost annual phenomenon in Ukraine caused by the significant human impact on the environment (Bessagnet et al., 2008; Goudie, 2009). Manifestations of wind erosion are widely observed on drained lands of Polessia.

Degradation processes directly related to the horizontal landscape mass transfer affect almost on $35 \%$ of the territory of Ukraine. The control of the process of horizontal landscape mass transfer requires optimization of the landscape structure. The effective implementation of such control is only possible if there are reliable, theoretically based, mathematical models of processes of horizontal landscape mass transfer (Van Oost et al., 2000; Barabanov, 2016).

A number of scientific publications describing different problems of mass transfer processes have appearedduring the last few decades. A vast range of engineering problems could be solved based on the reported solutions, for instance, some of the well-known problems are: subsurface mass transfer (Bonan, 1995; Prentice et al., 2007), mass transfer at the atmosphere boundary layer (Boardman and Favis-Mortlock, 1998; Arora, 2002; Hoek et al., 2008), pollution transfer across land and global water objects (Hendricks, 2006), transfer processes in chemical technology (Hritonenko and Yatsenko, 1999; Haggerty and Gorelick, 2006), mass transfer within the composite materials (Berselli et al., 2005; Djunin and Korzun, 2009).
The majority of the problems mentioned above can be divided into two main groups. The first group includes mass transfer problems within filtering materials where the influence of the environment structure on the transfer process is taken into account, and the mechanical influence of boundary surface on the transfer process is not taken into account. Such surface is considered only as a boundary of mass transfer domain, the boundary conditions of the corresponding mathematical problem are considered there (Bryant and Thompson, 2001; Capilla and Llopis-Albert, 2009). The second group is represented by the problems of mass transfer processes on a surface restricting the transfer domain (Dejch and Zarjankin, 1984; Kaimal and Finnigan, 1994; Djunin and Korzun, 2009). Here the influence of the boundary surface structure on the transfer process is taken into account. Additionally, the influence of porous environment structure and the averaging scale of transfer process are not taken into account.

Besides of the generalization mentioned, there is a specific group of problems which cannot be directly related to any of considered groups, such as mass transfer processes within vegetation in natural landscapes. The problem of landscape mass transfer is an important and urgent problem within environmental science. In particular, the problem of the interaction between the two-phase wind flow and landscape vegetation is a key to understanding evolution of landscape morphology, pollution distribution and soil erosion (Anderson and Wendt, 1995; Van Oost et al., 2000; Capilla and Llopis-Albert, 2009). In this context, the mathematical modeling of mass transfer processes within complex environments is an advanced and necessary tool for better understanding of environmental processes.

In this paper, we develop the formal framework to reflect correctly the problem of landscape mass transfer within the vegetation through a formal system with a reduced number of dependent 
variables and simplified boundary conditions. We develop a mathematical model of mass transfer process on an inhomogeneous boundary surface. The mechanical impact of boundary surface on the mass transfer process has been considered and taken into account. The mass transfer domain consists of a porous environment with a number of boundary surfaces. The mechanical impact of porous environment structure on mass transfer process has also been considered and taken into account. The substance source function has been developed here.

\section{Materials and methods}

The objectives of this research are: (1) to obtain a mathematical model for mass transfer on heterogeneous surfaces (including mechanical influence on the parameters of mass transfer), which is a border of the environment of mass transfer with the parameter characterizing the structure (and the model should consider the whole area of values of the structural parameter); (2) to describe the function of sources using the following mechanisms for material entering the flow of the carrier fluid (water, air): the dissolution of substance (physical-chemical process), rising into the flow of the solid phase through the normal pressure differential, mechanical influence onto the movement surface (mechanical interaction process of flow, boundary surface and mass transfer environment).

The object of the research is the processes of horizontal landscape mass transfer. The subject is mathematical modeling methods of the processes of horizontal landscape mass transfer.

The main methods used are the methods of analysis and system identification, methods of the theory of mathematical modeling, including all methodological aspects of stages of modeling, methods of set theory, graph theory, fluid mechanics, mathematical theory of filtration, statistical hydromechanics, methods of computational mathematics, programming.

\section{Results}

\section{Problem statement}

Transfer domain. Let's define the characteristics (conditions) of mass transfer domain.

(1) There is a certain environment existing in a whole mass transfer domain, additionally, such environment can be characterized by structural parameter, and exert a certain influence on mass transfer process: $\Omega=\left\{\Omega_{i}: n_{\Omega}=0 \ldots 1 ; i=1,2,3\right\}$.

(2) There are a set of boundary surfaces at the mass transfer domain; additionally, such surfaces have a certain structure influencing the mass transfer process: $\exists G \in \Omega, G=\left\{G_{i}: n_{G} \geq 0 ; i=1,2,3\right\}$. Besides the physical impact of set $G$ on mass transfer values realized through the structural parameter ${ }_{n_{G}}$ there are boundary conditions of corresponding mathematical problems of mass transfer on $\Omega_{i}$ defined at $G$.

(3) There is the set $F$ of substance source in the considered domain. The substance source is distributed on $\Omega$ :

$$
\begin{aligned}
& \exists F \in \Omega, F=\left\{f_{T}, f_{P}: f_{T, P}=f(\Omega, G) ; f_{T} \in G_{2} ; f_{P} \in G_{i}, \Omega_{i} ; i=2,3\right\} \\
& \text { where } f_{T} \text { is the set of solid substance source; } f_{P} \text { is the set of } \\
& \text { dissolved substance source. }
\end{aligned}
$$

(4) There is set $X$ existing in domain $\Omega$. And there are also mappings $g$ and ${ }_{h}$ defining the set ${ }_{C}$ of substance concentration and $F$ in $\Omega$ accordingly:

$$
\exists \mathrm{X} \in \Omega, g: \mathrm{X} \rightarrow C ; h: \mathrm{X} \rightarrow F ; C=\{c: c \in \Omega ; c=c(\Omega, G, F)\} .
$$

Conditions in $\Omega$. Let's consider the conditions necessary to solve the current problem.

(1) The turbulent type of motion. This can be accepted by considering the pulsations of velocity and mass concentration on the averaged transfer domain. The mechanism of such turbulence is completely considered in papers (Dejch and Zarjankin, 1984; Landau and Lifshitz, 2013). The turbulent motion is expressed in the equation through additional diffusive component ${ }_{D_{i}} \frac{\partial c}{\partial x_{i}}$ (Druzhinin and Shishkin, 1989; Berselli et al., 2005).

(2) Additional turbulence. Existence of such turbulence can be explained only by existence of $n_{\Omega} \neq 0$, which describes the porous environment structure. The possibility of turbulent filtration in the porous environment with certain values of $n_{\Omega}$ is considered in (El'Darov et al., 1996; Hendricks, 2006). $\Omega$ in the porous environment with different values of $n_{\Omega}$ the turbulence in the transfer domain can be explained both by the common reasons (Kaimal and Finnigan, 1994; Berselli et al., 2005; Djunin and Korzun, 2009), and by the existence of additional pulsations of velocity and substance concentrations generated by porous environment structure (Mil'kevich, 2003).

(3) Inhomogeneity of transfer domain. This condition is split into two separate conditions: inhomogeneity of the environment and inhomogeneity of boundary surfaces in the transfer domain. Inhomogeneity of the environment is due to instability of ${ }_{n_{\Omega}}$ within the averaging volume. This could be related to existence of periodic voids in a dense environment or consolidation of drops of filtering environment, there occur deviations (from the averaged values) of velocity and substance concentrations. Inhomogeneity of boundary surfaces in the transfer domain can be divided into two types.

(a) Vertical inhomogeneity. Such inhomogeneity is the cause of velocity and mass concentration pulsations as correlations with vertical parameters of boundary surfaces (for instance, slope drops, porous environment density drops, and micro-roughness).

(b) Horizontal (spatial) inhomogeneity. Such inhomogeneity is the cause of velocity and mass concentration deviations from the average values in the average domain. Spatial inhomogeneity relates to the number of flow lines crossing in the transfer domain. This can be caused by significant changes in the flow parameters.

(4) Scale of averaging. The information mentioned in (Berselli et al., 2005) is about the necessity of corrections in the mass transfer equation for inhomogeneous environments. These corrections are related to the influence of averaging scale. Increasing the averaging scale in an inhomogeneous domain can cause the higher inhomogeneity degree and respectively can cause a higher degree of local deviations of velocity and mass concentrations from the averaged value. Considering the dissemination of averaging scale corrections to the considering inhomogeneous transfer domain $\Omega$ is a reasonable correction in this case.

(5) The possibility of limit values acquired by structural parameter $n_{\Omega, G} \rightarrow 0,1$. Taking into account such structural parameter values, we can consider the range of physical processes (occurring in ${ }_{G}$ ) in the same mathematical model. For example, mass transfer modeling with consideration of $G$ permeable and impermeable obstacles and macro-roughness.

Thus, the problem of mathematical modelling of mass transfer process on inhomogeneous surface in porous environments can be defined as following. The problem solution is to define the mappings $g$ and ${ }_{h}$ in $\Omega$ by considering and taking into account properties (1-4), mass transfer conditions in $\Omega$ and applying conservation equation (1)

$$
\frac{\partial c}{\partial t}+\nabla \cdot(c v)=f
$$

(Dejch and Zarjankin, 1984; Djunin and Korzun, 2009).

\section{Mass transfer equation}

To determine the average values in equation (1) the averaged and momentary values of velocity and mass concentration have to be considered. Time-averaging and volume-averaging operations should be provided at the same time by the following mode (Djunin and Korzun, 2009):

$$
\langle c\rangle=\frac{1}{2 \Omega_{A} \Delta t} \int_{\Omega_{A}} \int_{2 \Delta t} c \cdot d \Omega_{A} d t
$$




$$
\langle v\rangle=\frac{1}{2 \Omega_{A} \Delta t} \int_{\Omega_{A}} \int_{2 \Delta t} v \cdot d \Omega_{A} d t
$$

where $\langle c\rangle,\langle v\rangle$ - time and volume averaged values of mass concentration and velocity accordingly; $\Omega_{A}-$ the volume filled by substance with concentration $c$; $t$ - time.

Considering (2) and (3) we have the following expressions

$$
c=\langle c\rangle+c^{\prime}, v=\langle v\rangle+v^{\prime} \text {. }
$$

Momentary (pulsation) component in (4) can be presented as a set of statistically independent pulsations existing due to the action of statistically independent events between which there are no correlation links.

$$
c=c_{1}^{\prime}+c_{2}^{\prime}+c_{3}^{\prime}, \quad v=v_{1}^{\prime}+v_{2}^{\prime}+v_{3}^{\prime} ;
$$

where $c_{1}^{\prime}, v_{1}^{\prime}$ - pulsation components of mass concentration and velocity accordingly; such pulsations are generated by the common turbulence process (condition 1); $c_{2}^{\prime}, v_{2}^{\prime}$ - pulsation components of mass concentration and velocity accordingly; such pulsations are generated by the interaction process between flow and porous environment structure (condition 2); $c_{3}^{\prime}, v_{3}^{\prime}$ - pulsation components of mass concentration and velocity accordingly; such pulsations are generated by interaction process between flow and vertical inhomogeneity of boundary surfaces (condition 3a).

Taking into account the average values properties (Dejch and Zarjankin, 1984; Djunin and Korzun, 2009) and (4, 5) we obtain the following:

$$
\langle c v\rangle=\left\langle\left(\langle c\rangle+c_{1}^{\prime}+c_{2}^{\prime}+c_{3}^{\prime}\right)\left(\langle v\rangle+v_{1}^{\prime}+v_{2}^{\prime}+v_{3}^{\prime}\right)\right\rangle=\langle c\rangle\langle v\rangle+\left\langle c_{1}^{\prime} v_{1}^{\prime}\right\rangle+\left\langle c_{1}^{\prime} v_{2}^{\prime}\right\rangle+\left\langle c_{1}^{\prime} v_{3}^{\prime}\right\rangle+
$$$$
+\left\langle c_{2}^{\prime} v_{1}^{\prime}\right\rangle+\left\langle c_{3}^{\prime} v_{1}^{\prime}\right\rangle
$$

There are only six components at the right side of (6). This can be explained by the following. The components statistical independence in (5) and also the statistical independence of events generating such components create the one-side correlation link between from one side $c_{2}^{\prime}, v_{2}^{\prime}$ and $c_{3}^{\prime}, v_{3}^{\prime}$, and from another side $c_{1}^{\prime}, v_{1}^{\prime}$. There is no correlation link between $c_{2}^{\prime}, v_{2}^{\prime}$ and $c_{3}^{\prime}, v_{3}^{\prime}$.

The volume-averaging properties are used to define necessary deviations of mass concentration and velocity. As a result of the volume averaging we have the following (Capilla and Llopis-Albert, 2009):

$$
\langle c\rangle\langle v\rangle=\left\langle c\left(x_{0}, t\right)\right\rangle\left\langle v\left(x_{0}, t\right)\right\rangle+\langle(\tilde{c}(x, t)+\delta \tilde{c})(\tilde{v}(x, t)+\delta \tilde{v})\rangle,
$$

where $c\left(x_{0}, t\right)$ and $v\left(x_{0}, t\right)$-averaged values of concentration and velocity accordingly at the point $x_{0}$ which is the center of mass of averaging volume; $\tilde{c}(x, t)$ and $\tilde{v}(x, t)$-deviations of local values of concentration and velocity accordingly in point $x$, from their average values in this point. These values are conditioned by inhomogeneity of transfer domain and increase with a higher inhomogeneity degree of transfer domain; $\delta \tilde{c}$ and $\delta \tilde{v}$-difference between values $\langle c, v\rangle$ at the points ${ }_{x_{1}}$ and ${ }_{x_{0}}$ accordingly. The $\delta \tilde{c}$ and $\delta \tilde{v}$ are conditioned by the averaging scale and increase with a higher averaging scale.

Additional deviations can be defined as sets of statistically independent deviations conditioned by porous environment and boundary surfaces properties of transfer domain.

$$
\tilde{c}=\tilde{c}_{2}+\tilde{c}_{3}, \tilde{v}=\tilde{v}_{2}+\tilde{v}_{3}, \delta \tilde{c}=\delta \tilde{c}_{2}+\delta \tilde{c}_{3}, \delta \tilde{v}=\delta \tilde{v}_{2}+\delta \tilde{v}_{3},
$$

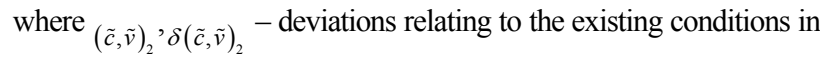
the porous environment; $(\tilde{c}, \tilde{v})_{3}{ }^{\prime} \delta(\tilde{c}, \tilde{v})_{3}$ - deviations relating to the existing conditions on boundary surfaces.

In the first approach we assume that applied couples $\tilde{c}$ and $\delta \tilde{v}$, $\delta \tilde{c}$ and $\tilde{v},{ }_{(\tilde{c}, \tilde{v})_{i}}$ and ${ }_{(\tilde{c}, \tilde{v})_{j}}{ }^{\delta} \delta(\tilde{c}, \tilde{v})_{i}$ and ${ }_{\delta(\tilde{c}, \tilde{v})_{j}}$ are statistically independent. Considering (7) and (8) equation (6) can be transformed into:

$\langle c v\rangle=\left\langle c\left(x_{0}, t\right)\right\rangle\left\langle v\left(x_{0}, t\right)\right\rangle+\left\langle\tilde{c}_{2} \tilde{v}_{2}\right\rangle+\left\langle\tilde{c}_{3} \tilde{v}_{3}\right\rangle+\left\langle\delta \tilde{c}_{2} \delta \tilde{v}_{2}\right\rangle+\left\langle\delta \tilde{c}_{3} \delta \tilde{v}_{3}\right\rangle+$, $+\left\langle c_{1}^{\prime} v_{1}^{\prime}\right\rangle+\left\langle c_{1}^{\prime} v_{2}^{\prime}\right\rangle+\left\langle c_{1}^{\prime} v_{3}^{\prime}\right\rangle+\left\langle c_{2}^{\prime} v_{1}^{\prime}\right\rangle+\left\langle c_{3}^{\prime} v_{1}^{\prime}\right\rangle$
Rewrite (1) considering (9), due to pulsations $c_{3}^{\prime}$ and $v_{3}^{\prime}$ are changeable in an averaging domain, the components $\left\langle c_{1}^{\prime} v_{3}^{\prime}\right\rangle$ and $\left\langle c_{3}^{\prime} v_{1}^{\prime}\right\rangle$ are considered as neglected:

$\frac{\partial\langle c\rangle}{\partial t}+\nabla \cdot(\langle c\rangle\langle v\rangle)=-\nabla \cdot\left(\left\langle\tilde{c}_{2} \tilde{v}_{2}\right\rangle+\left\langle\tilde{c}_{3} \tilde{v}_{3}\right\rangle+\left\langle\delta \tilde{c}_{2} \delta \tilde{v}_{2}\right\rangle+\left\langle\delta \tilde{c}_{3} \delta \tilde{v}_{3}\right\rangle+\right.$

$\left.+\left\langle c_{1}^{\prime} v_{1}^{\prime}\right\rangle+\left\langle c_{1}^{\prime} v_{2}^{\prime}\right\rangle+\left\langle c_{2}^{\prime} v_{1}^{\prime}\right\rangle\right)+\langle f\rangle$

There are seven additional components in equation (10). These components in some way have to be linked to averaged values of concentration and velocity. These components can be defined within the following expressions:

$$
\begin{aligned}
& -\left\langle c_{1}^{\prime} v_{i}^{\prime}\right\rangle=D_{i} \frac{\partial\langle c\rangle}{\partial x_{i}},(\text { Berselli et al., 2005), } \\
& -\left\langle c_{2}^{\prime} v_{i}^{\prime}\right\rangle=D_{2_{c^{i}}} \frac{\partial\langle c\rangle}{\partial n_{i}}, \\
& -\left\langle c_{1}^{\prime} v_{2}^{\prime}\right\rangle=D_{2_{V} i} \frac{\partial\langle v\rangle}{\partial n_{i}} \\
& -\left\langle\tilde{c}_{2} \tilde{v}_{2}\right\rangle=D_{2 i}^{\prime} \frac{\partial\langle c\rangle}{\partial n_{i}}, \\
& -\left\langle\tilde{c}_{3} \tilde{v}_{3}\right\rangle=D_{3 i}^{\prime} \frac{\partial\langle c\rangle}{\partial x_{i}} \\
& -\left\langle\delta \tilde{c}_{3} \delta \tilde{v}_{3}\right\rangle=-\frac{\partial\langle c\rangle}{\partial x_{i}} \frac{\partial\langle v\rangle}{\partial x_{j}}\left[\left(x_{i \lambda_{0}}-x_{i \lambda}\right)\left(x_{j \lambda_{0}}-x_{j \lambda}\right)\right]=D_{3 i}^{\prime \prime} \frac{\partial\langle c\rangle}{\partial x_{i}} \\
& -\left\langle\delta \tilde{c}_{2} \delta \tilde{v}_{2}\right\rangle=-\frac{\partial\langle c\rangle}{\partial n_{i}} \frac{\partial\langle v\rangle}{\partial n_{j}}\left[\left(x_{i n_{0}}-x_{i n}\right)\left(x_{j n_{0}}-x_{j n}\right)\right]=D_{2 j}^{\prime \prime} \frac{\partial\langle c\rangle}{\partial n_{i}},
\end{aligned}
$$

where ${ }_{D_{i}}$ - diffusion coefficient; ${ }_{D_{k i}}{ }^{\prime} D_{k i}^{\prime}, D_{k i}^{\prime \prime}-$ mechanical dispersion coefficient, $k=2,3,2 c, 2 v ; n$ - porosity.

The physical meaning of velocity gradient $\frac{\partial\langle v\rangle}{\partial n}$ is described in paper (Mil'kevich, 2003). Concentration gradient $\frac{\partial\langle c\rangle}{\partial n}$ has the similar nature.

To obtain (12-17) the following assumption has been applied. Correlations between pulsation components and deviation components are directly proportional to corresponding gradients; also Taylor series have been applied to functions $\langle v(x, t)\rangle,\langle c(x, t)\rangle$ together with $x_{\lambda_{0}}$ (the central point of the averaging subdomain $\Omega_{i}^{\lambda} \in \Omega_{i}$ ) and $x_{n_{0}}$ (the central point of the averaging subdomain $\Omega_{i}^{n} \in \Omega_{i}$ ) and the linear components of series are left.

Rewrite (1), using (11-17):

$$
\begin{aligned}
& \frac{\partial\langle c\rangle}{\partial t}+\nabla \cdot(\langle c\rangle\langle v\rangle)=\nabla \cdot\left[\left(D_{i}+D_{3 i}^{\prime}+D_{3 i}^{\prime \prime}\right) \frac{\partial\langle c\rangle}{\partial x_{i}}+\left(D_{2 c i}+D_{2 i}^{\prime}+D_{2 i}^{\prime \prime}\right) \frac{\partial\langle c\rangle}{\partial n_{i}}+D_{2 r i} \frac{\partial\langle v\rangle}{\partial n_{i}}\right]+ \\
& +\langle f\rangle
\end{aligned}
$$

To complete the model, the boundary conditions of the first, the second and the third types have to be defined (Druzhinin and Shishkin, 1989; Berselli et al., 2005; Djunin and Korzun, 2009). Depending on the character of transfer subdomain and particular conditions, different kinds of mathematical problems can be formulated based on the model (18).

\section{Mass source function}

In the first approach the following assumption can be accepted. Sorption and desorption processes are considered as the main physical-chemical processes responsible for substance transfer from source to flow and from flow back to source. Such processes can be described in the following way (Berselli et al., 2005):

$$
f_{p}^{\prime}=D_{p}\left(c-c_{S}\right),
$$

where $f_{p}^{\prime}$ - function defining the physical-chemical processes of substance transfer up to flow, $\left[\frac{g}{m^{2} \cdot s}\right] ; D_{p}-$ proportional coefficient, 
$\left[\frac{m}{s}\right] ; c$ - substance concentration in the flow, $\left[\frac{\mathrm{g}}{\mathrm{m}^{3}}\right] ; c_{S}$ - substance concentration in the phase split surface, $\left[\frac{\mathrm{g}}{\mathrm{m}^{3}}\right]$.

For (19) we can write:

$$
f_{p}^{\prime}= \begin{cases}\left(c-c_{S}\right)>0, D_{p}=D_{p 1} & \text { a }) ; \\ \left(c-c_{S}\right) \leq 0, D_{p}=D_{p 2} & \text { b }),\end{cases}
$$

where (a) - adsorption process, (b) - desorption process.

Considering dimensions of the units mentioned above, the following statement should be noted. If we accept the velocity dimension for ${ }_{D_{p}}$ (this can be explained as velocity of directional molecular-diffusion motion of substance) it is possible to use (19), which can be applied for the whole inhomogeneous domain.

We present it in the following way:

$$
f_{p}^{\prime \prime}=D_{p}\left(c-c_{S}\right) S_{\Omega},
$$

where $f_{p}^{\prime \prime}$ - substance discharge through the split phase surface, $\left[\frac{g}{s}\right]$; $S_{\Omega}$ - the square of split phase surface, $\left[\mathrm{m}^{2}\right]$.

Let's show $S_{\Omega}$ as a sum of two components - the square of split phase surface on set ${ }_{G_{i}}$ and the square of split phase surface on set $\Omega_{i}$ : $S_{\Omega}=S_{G_{i}}+S_{\Omega_{i}}$. It's too complicated to define the values ${ }_{S_{G_{i}}}, S_{\Omega_{i}}$ exactly. So in this case we assume the following: $S_{G_{i}}, S_{\Omega_{i}}$ are proportionally dependant on some parameters in ${ }_{G_{i}}$ and $\Omega_{i}$ :

$$
S_{G_{i}}=\alpha \frac{h}{l}, S_{\Omega_{i}}=\beta\left(1-\frac{V_{n}}{V}\right),
$$

where ${ }_{\alpha, \beta}$ - proportional coefficients, $\left[\mathrm{m}^{2}\right] ; h$ - the average height of micro-roughness in ${ }_{G_{i}},[m], l-$ the average distance between units of micro-roughness in ${ }_{G_{i}},[\mathrm{~m}], V_{n}$ - the environment volume not filled by a solid phase, $\left[m^{3}\right] ; V-$ the whole volume of the environment $\left[\mathrm{m}^{3}\right]$.

Denoting $g=\frac{h}{l}$ and $\omega=\left(1-\frac{V_{n}}{V}\right)$, we write (20) in following way:

$$
f_{p}^{\prime \prime}=D_{p} \beta\left(c-c_{S}\right)\left(\frac{\alpha}{\beta} g+\omega\right) .
$$

Such form for $f_{p}^{\prime \prime}$ is convenient because component $\left(\frac{\alpha}{\beta} g+\omega\right)$ is dimensionless, but ${ }_{D_{p} \beta}$ can be defined as a new diffusion coefficient with the following dimension $\left[\frac{m^{3}}{s}\right]$. The new diffusion coefficient might be defined by the empirical investigation.

The averaging operation has the form presented bellow:

$\left\langle f_{p}^{\prime \prime}\right\rangle=\frac{1}{2 \Omega_{A} \Delta t} \int_{\Omega_{A}} \int_{2 \Delta t} D_{p} \beta\left(c-c_{s}\right)\left(\frac{\alpha}{\beta} g+\omega\right) d \Omega_{A} d t=\left[D_{p} \beta\left(c-c_{s}\right)\left(\frac{\alpha}{\beta} g+\omega\right)\right]_{c e p}$

For $f_{p}^{\prime}$ the averaged value is described in the same way.

To obtain the solid substance source function, there have been used the results presented in papers (Djunin and Korzun, 2009; Mil'kevich, 2003).

The double phase flow equation (both for solid and liquid phases) for considering the transfer domain has the following form:

$$
\begin{aligned}
& \rho(1-s)(\bar{V} \cdot \nabla) \bar{V}+\rho_{s} s\left(\overline{V_{s}} \cdot \nabla\right) \overline{V_{s}}+\rho\left[\bar{V}\left(\nabla \cdot K_{w_{11}}+\nabla \cdot K_{w_{12}}+\nabla \cdot K_{w_{21}}\right)\right]- \\
& -\rho_{s}\left[\overline{s_{s}}\left(\nabla \cdot K_{s_{11}}+\nabla \cdot K_{s_{12}}+\nabla \cdot K_{s_{21}}\right)\right]=\rho(1-s) g+\rho_{s} s g-\nabla \cdot \Pi- \\
& -\rho\left[\nabla \cdot T_{11}+\nabla \cdot T_{12}+\nabla \cdot T_{21}\right]-\rho_{s}\left[\nabla \cdot T_{s_{11}}+\nabla \cdot T_{s_{12}}+\nabla \cdot T_{s_{21}}\right]- \\
& -\rho\left[A \bar{V}+B V^{2}-\frac{B}{\rho}\left(\sigma_{11 i}+2 \sigma_{12 i}\right)\right]
\end{aligned}
$$

where $\rho, \rho_{s}-$ density of the liquid and the solid phase of flow accordingly, $\left[\frac{g \cdot s^{2}}{m^{4}}\right] s$ - solid phase concentration; ${ }_{\bar{v}, \bar{v}_{s}}$ - averaged velocity of the liquid and the solid phase of flow accordingly, $[\mathrm{m}]$; $\nabla$ - Hamilton operator; $\bar{K}_{w}, \bar{K}_{s}$ - pulsation vectors of the liquid and the solid phase of flow accordingly; $\bar{g}, \bar{\varepsilon}, \bar{\varepsilon}_{s}-$ gravity acceleration and non-gravity forces accelerations of the liquid and the solid phase of flow accordingly, $\left[\frac{\mathrm{m}}{\mathrm{s}^{2}}\right] ; \Pi$ - molecular strain tensor; $\mathrm{T}_{11}, \mathrm{~T}_{s 11}-$ averaged tensors of additional strains caused by mixing of liquid and solid particles; $\mathrm{T}_{12}, \mathrm{~T}_{21}, \mathrm{~T}_{s 12}, \mathrm{~T}_{s 21}$ - averaged tensors of additional strains caused by the interaction between flow and porous media structure; $\sigma_{11 i}, \sigma_{12 i}-$ normal turbulence strains;

$$
A=\alpha{\frac{(1-n)^{2}}{d^{2}\left(n-\dot{c}_{0}\right)^{2}}}^{\prime} B=\beta \cdot{\frac{1-n}{d\left(n-\dot{c}_{0}\right)}}^{\prime},
$$

where $d$ - units thickness of roughness; $n$ - porosity; ${ }_{\left(n-\dot{c}_{0}\right)}-$ effective porosity; $\alpha, \beta$ - proportional coefficients.

We consider a flat, quasi-steady, parallel to horizontal plane $y=0$ and homogeneous along the $x$-axis flow. We provide the mapping of equation (22) to the $y$-axis, but consider the following

$\mathrm{i}_{\frac{\partial \Pi_{3 k}}{\partial x_{k}}}=\frac{\partial T_{3 k}}{\partial x_{k}}=\frac{\partial T_{S_{3 k}}}{\partial x_{k}}=0$, with $k=1,2$. We obtain the following

$$
\rho(1-s) g+\rho_{s} s g=\frac{\partial}{\partial y}\left[\Pi_{y y}+\rho\left(T_{11 y y}+T_{12, y y}+T_{21, y y}\right)\right]+\rho_{s}\left(T_{s 1, y,}+T_{s i 2, y}+T_{s 21, y}\right)+
$$

$+B\left(T_{11, y y}+T_{12, y y}+T_{21, y y}\right)$.

We integrate (23) in $y$ :

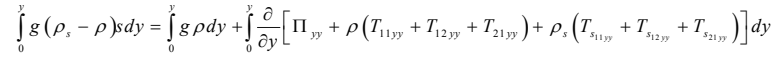

$$
\begin{aligned}
& +B \int\left[\left(T_{11, y y}+T_{12, y y}+T_{21 y y}\right)\right] d y
\end{aligned}
$$

We denote $\Pi(y)$ as normal molecular strain drop in the layer $0 \div y$ without considering pulsations in the liquid and the solid phases: $\Pi(y)=\Pi_{0}-\Pi_{y y}-g \rho y \cdot$

We denote

$$
\int_{0}^{y} g\left(\rho_{s}-\rho\right) s d y \text { as shown in (Djunin and Korzun, }
$$

2009) through $j(y)$, which is a flow load.

Thereby, the condition for solid particles rising is

$$
j(y)=\Pi(y)-\left[\left(\rho+B_{y}\right)\left(T_{11 y y}+T_{12 y y}+T_{21 y y}\right)+\rho_{s}\left(T_{s_{11, y}}+T_{s_{12, y}}+T_{s_{21, y}}\right)\right]>0
$$

In order to analyze the rough influence on solid particles rising value ${ }_{j(y)}$, the value $j_{1}(y)$ for a flow which is not moving through the roughness and value $j_{2}(y)$ for a flow which is moving through the roughness, have to be compared.

We define ${ }_{j_{1}(y)}$ in the following way (Djunin and Korzun, 2009):

$$
j_{1}(y)=\Pi\left(x_{3}\right)-T_{F}(y)>0,
$$

where $T_{F}(y)$ - the additional value of normal pressure caused by diagonal components of pulsation tensions, $T_{F}(y)=\rho T_{y y}+\rho_{s} T_{s_{y y}}$.

The second component of the right side of expression (24) is presented as:

$$
\left(\rho+B_{y}\right)\left(T_{11 y y}+T_{12 y y}+T_{21 y y}\right)+\rho_{s}\left(T_{s_{11 y y}}+T_{s_{12 y}}+T_{s_{21, y}}\right)=T_{F}(y)+T_{H}(y),
$$
where

$$
T_{H}(y)=\left(\rho+B_{y}\right)\left(T_{12 y y}+T_{21, y y}\right)+B_{y} T_{11 y y}+\rho_{s}\left(T_{s_{12, y}}+T_{s_{21, y}}\right)^{.}
$$

Expression (24) is transformed to

$$
j_{2}(y)=\Pi(y)-T_{F}(y)-T_{H}(y)>0
$$

Considering the same flow $j_{1}(y)$ is a flow load before moving through the roughness layer and $j_{2}(y)$ is a flow load during the motion through the roughness layer, thus $j_{1}(y)>j_{2}(y)$. Normal turbulent strains $T_{T_{H}(y)}$ caused by roughness, reduce the flow loads.

The extreme value of discharge rates for the solid phase during motion in $\Omega$ has to be defined. We use the expression (26). Since the 
solid phase discharge rates have to be taken into account, we transform $j_{2}(y)$ as it is shown below:

$j_{2}(y)=\int_{0}^{y} g\left(\rho_{s}-\rho\right) d y=\left(1-\frac{\rho}{\rho_{s}}\right) \int_{0}^{y} \rho_{s} s g d y=\left(1-\frac{\rho}{\rho_{s}}\right) j_{2_{s}}(y)$

We consider, as in (Djunin and Korzun, 2009), that normal pressure drop is caused by the vortex layer presented in the transfer domain. Considering the certain environment, where the structural parameter value is not a zero, the rate of normal pressure drop is going to be decreased. We rewrite (26) considering everything mentioned above and (27):

$$
j_{2_{s}}(y)=\frac{1}{\left(1-\frac{\rho}{\rho_{s}}\right)}\left(\Pi(y)-T_{F}(y)-T_{H}(y)\right)=\frac{a_{s} \rho}{2\left(1-\frac{\rho}{\rho_{s}}\right)}\left[\left.l_{s} \frac{\partial u}{\partial y}\right|_{\text {cep }}\right]^{2}
$$

The velocity gradient on the surface ${ }_{y=0}$ can be presented as averaged velocity gradient of flow through the proportional coefficient $\xi$ (Djunin and Korzun, 2009):

$$
\left.\xi \cdot \frac{\partial u}{\partial y}\right|_{0}=\left.\frac{\partial u}{\partial y}\right|_{c e p}
$$

Can be written, (Djunin and Korzun, 2009):

$$
\left.\frac{\partial u}{\partial y}\right|_{0}=\frac{\tau_{0 s}}{\rho v\left(1+c_{0}\right)}
$$

under condition $y=0$.

From (Mil'kevich, 2003), due to some transformations we obtain:

$$
\frac{\tau_{0 s}}{\rho}=\frac{\sqrt{\pi} v\left(1+c_{\tau}\right) \Delta u}{2 \delta_{e} \sqrt{\ln \frac{h}{\delta_{e}}}-\frac{8}{5} \psi \delta_{e}^{2}},
$$

where $\tau_{0 s}$-tangent strain on boundary surface; $\delta_{e}$ - effective roughness, $\left(1+c_{\tau}\right) c_{s} \delta=\delta_{e} ; \quad c_{s}=\sqrt{\frac{\tau_{0} k}{\tau_{0 s}}} \frac{a}{a_{s}} ; \quad c_{\tau}=\frac{2}{\sqrt{\pi}} \cdot \frac{\int_{0}^{\phi_{s}} c \cdot e^{-\phi_{2 s}} d \phi_{2_{s}}}{\operatorname{erf} \phi_{2_{s}}} \quad c-$ function depending on solid phase concentration in flow; $\psi$ - parameter depending on the environment structure (Mil'kevich, 2003); $\phi_{2 S}=\frac{a_{S} u(y)}{v_{*}}+\frac{2}{5} \frac{\psi(n)}{v_{*} a_{S}} ; a_{S}-$ proportional coefficient; $v_{*}-$ dynamical velocity.

Using (29-31), we rewrite (28) in the following way:

$$
j_{2_{s}}(y)=\frac{a_{s} l_{s}^{2} \xi^{2}\left(u_{h}-u_{k p h}\right)^{2} \pi}{2\left(\frac{1}{\rho}-\frac{1}{\rho_{s}}\right) \cdot\left(\left(2 c_{s} \delta \sqrt{\ln \frac{h}{\delta_{e}}}-\frac{8}{5} \psi c_{s}^{2} \delta^{2}\left(1+c_{\tau}\right)\right)\left(1+c_{0}\right)\right)^{2}} \equiv f_{T}^{\prime},
$$

where ${ }_{u_{h}}$ - velocity at height $h ;{ }_{u_{k p h}}$ - critical velocity at height ${ }_{h} ; f_{T}^{\prime}-$ function defining the processes of solid substance transfer up to flow, $\left[\frac{g}{m^{2} \cdot s}\right]^{;} f_{T}^{\prime \prime}=f_{T}^{\prime} \cdot S_{G_{i}}=f_{T}^{\prime} \cdot \alpha \cdot g$, where $f_{T}^{\prime \prime}-$ solid substance discharge through the surface ${ }_{S_{G_{i}}}\left[\frac{g}{s}\right]$. The way to obtain the averaged value for $f_{T}^{\prime}$ and $f_{T}^{\prime \prime}$ is the same as for $\left\langle f_{p}^{\prime \prime}\right\rangle$.

4. Numerical simulation of two-phase wind flow interaction with landscape vegetation. The results of numerical simulation of two-phase wind flow through the landscape vegetation are shown in Fig. 1. Vertical distribution of the dense phase of the flow is shown in Fig. 2.

\section{Discussion}

In the scientific literature a number of papers describe various problems of mass transfer. They solve a wide range of problems in connection with different types of technical problems: the problem of underground mass transfer, transfer of pollutants in the atmosphere, spreading of pollution in water bodies, the problem of chemical engineering, mass transfer in composite materials etc. (Haggerty and Gorelick, 1998; Berselli et al., 2005; Djunin and Korzun, 2009).
All tasks solving these problems can be divided into two groups. The first group includes the problems of mass transfer in the filter material taking into account the influence of the structure of the environment on the parameters of mass transfer and not taking into account the mechanical stress of the boundary surface of movement on the parameters of mass transfer (this surface is considered as a border region of mass transfer only, which specifies the boundary conditions for the corresponding mathematical task): underground mass transfer - the structure of the environment and the averaging scale are considered, mass transfer occurs by diffusion, mechanical dispersion and convection (often there are tasks which do not take into account the environment and convection transfer) (Kaimal and Finnigan, 1994; Boardman and Favis-Mortlock, 1998; Prentice et al., 2007); mass transfer in nonporous composite materials - a probabilistic character of distribution of the environment heterogeneities, mass transfer due to diffusion are considered (Anderson and Wendt, 1995; Arora, 2002). The second group includes problems of mass transfer to surfaces, which restricts the region of mass transfer taking into account surface structure on the parameters of mass transfer not taking into the impact of averaging scale of inhomogeneous surfaces and structures of the environment in the region of mass transfer on the parameters of mass transfer: mass transfer in industrial processes that use multiphase flows, such as transfer of dispersed liquid phase by gas flow above the surface of the turbulent fluid flow - the influence of the surface of the turbulent flow on the parameters of gas flow (carrier phase) over this surface and the role of the surface in the activity of material sources, evenly distributed over the surface are considered (Bryant and Thompson, 2001); the spread of pollutants in the atmosphere, the influence of the marginal boundary on the flow parameters is considered (changes in flow rate of the carrier phase), mass transfer through the processes of diffusion and convection transfer (Haggerty and Gorelick, 1998); similar approaches in the tasks concerning spreading of pollution in water bodies (Van Oost et al., 2000), the problem of soil erosion (Boardman and Favis-Mortlock, 1998; Van Oost et al., 2000).

Notably, this group of tasks is also characterized by the fact that the mechanical influence of the boundary surface on the mass transfer parameters is considered mainly due to the speed parameter that appears only formal in equations of motion of the carrier phase and is not directly reflected in the equations of mass transfer.

In terms of the abovementioned models there are problems in our model, the formal setting of which does not strictly belong to these groups of tasks - this is the task of horizontal landscape mass transfer. Within the problem of the nature of the process and the field of mass transfer, we have included in one model the conditions of mass transfer inherent in both groups of tasks (simultaneously taking into account the surface and structure of the environment of mass transfer) as well as specific processes occurring in the field of mass transfer and not included in the already known models. It is characteristic for this field of mass transfer is that by changing the values of model parameters you can naturally go to the models of these groups of tasks without changing the structure of the original model.

\section{Conclusions}

A mathematical model describing the processes of mass transfer on inhomogeneous surface in the porous environment was developed and theoretically investigated in this paper. The mechanical impact of boundary surfaces and porous environment structure on a mass transfer process was considered and included into the model system. Also the mass source function adapted to the specific inhomogeneous domain was developed and investigated. 

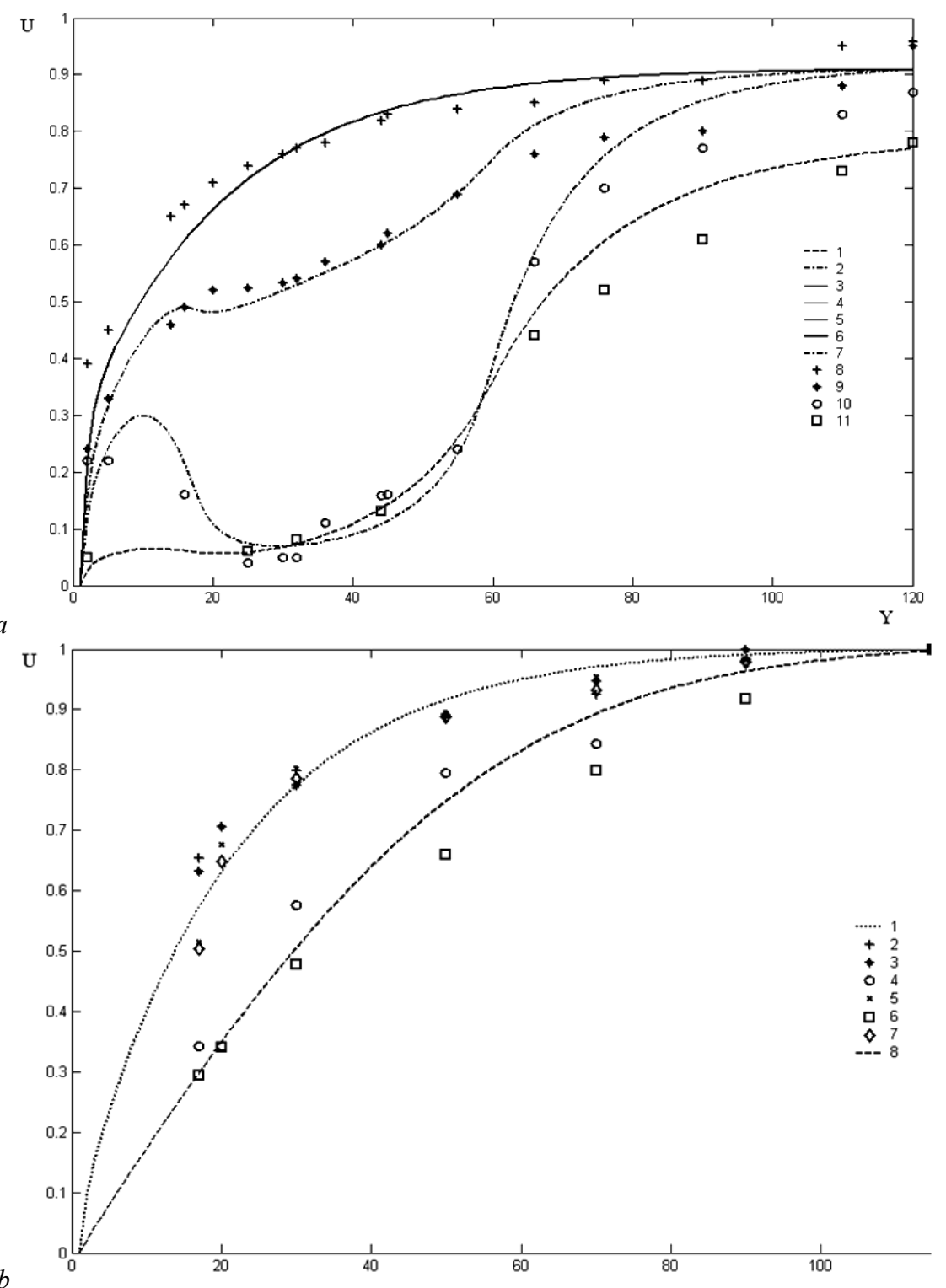

Fig. 1. The results of numerical simulation of two-phase wind flow through landscape vegetation: the points - empirical data; lines - simulation (wind flow interacts with a forest); $a$ - velocity profiles before and during the flow interaction with a forest; $b$ - velocity profiles of "clean" and two-phase flow

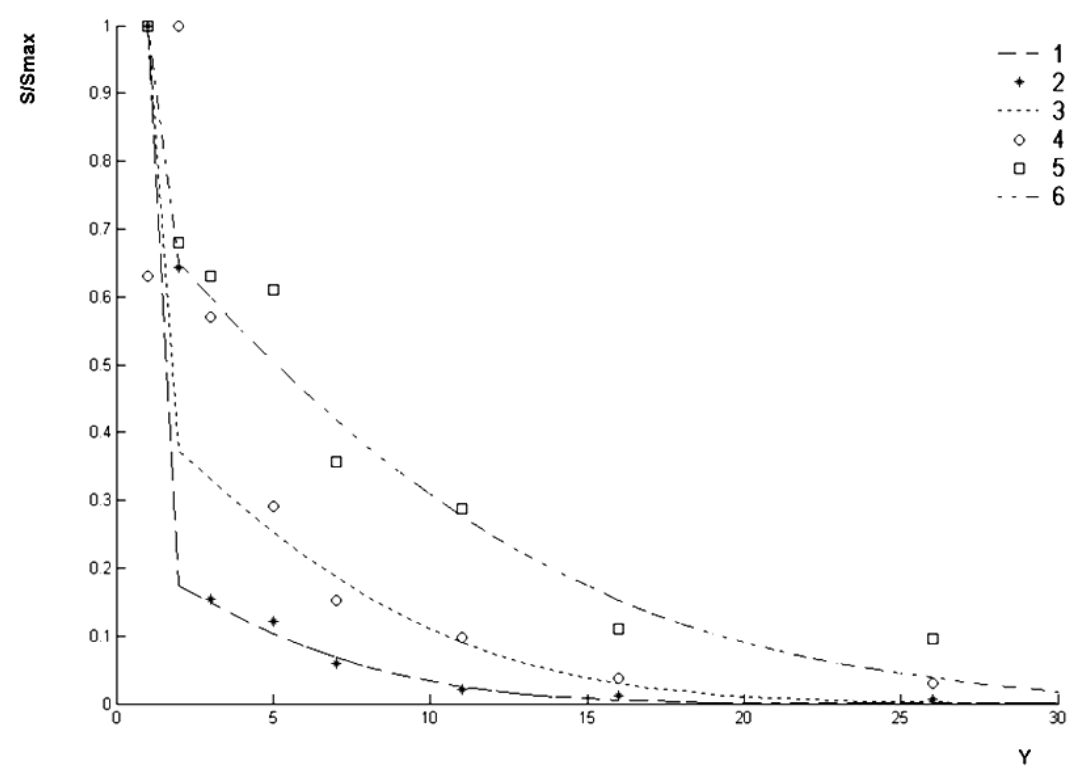

Fig. 2. Vertical distribution of the dense phase of the flow: points - empirical data; lines - simulation 


\section{References}

Anderson, J. D., \& Wendt, J. (1995). Computational fluid dynamics (Vol. 206). McGraw-Hill, New York.

Arora, V. (2002). Modeling vegetation as a dynamic component in soil vegetation - atmosphere transfer schemes and hydrological models. Reviews of Geophysics, 40(2).

Barabanov, A. T. (2016). Principles of adaptive-landscape generation and development of soil protection agricultural systems. Geography and Natural Resources, 37(2), 106-113.

Berselli, L., Iliescu, T., \& Layton, W. J. (2005). Mathematics of large eddy simulation of turbulent flows. Springer Science and Business Media.

Bessagnet, B., Menut, L., Aymoz, G., Chepfer, H., \& Vautard, R. (2008). Modeling dust emissions and transport within Europe: The Ukraine March 2007 event. Journal of Geophysical Research: Atmospheres (1984-2012), 113(D15).

Bonan, G. B. (1995). Land-atmosphere interactions for climate system models: coupling biophysical, biogeochemical, and ecosystem dynamical processes. Remote Sensing of Environment, 51(1), 57-73.

Bryant, S. L., \& Thompson, K. E. (2001). Theory, modeling and experiment in reactive transport in porous media. Current Opinion in Colloid and Interface Science, 6(3), 217-222.

Capilla, J. E., \& Llopis-Albert, C. (2009). Gradual conditioning of nonGaussian transmissivity fields to flow and mass transport data: 1 . Theory. Journal of Hydrology, 371(1), 66-74.

Dejch, M. E., \& Zarjankin, A. E. (1984). Gidrogazodinamika [Fliud dinamics]. Energoatomizdat, Moscow (in Russian).

Djunin, V. I., \& Korzun, A. V. (2009). Role of regional infiltration recharge sources in the formation of deep fluids and petroliferous basin hydrodynamic zoning. In: Hydrogeodynamics of Oil and Gas Basins Springer Netherlands. pp. 37-45.

Druzhinin, N. I., \& Shishkin, A. I. (1989). Matematicheskoe modelirovanie i prognozirovanie zagrjaznenija poverhnostnyh vod sushi. Gidrometeoizdat, Leningrad (in Russian)
El’Darov, E. G., Mamedov, F. V., Gol'Dberg, V. M., \& Zaikov, G. E. (1996). A kinetic model of polymer degradation during extrusion. Polymer Degradation and Stability, 51(3), 271-279.

Goudie, A. S. (2009). Dust storms: Recent developments. Journal of Environmental Management, 90(1), 89-94.

Haggerty, R., \& Gorelick, S. M. (1998). Modeling mass transfer processes in soil columns with pore-scale heterogeneity. Soil Science Society of America Journal, 62(1), 62-74.

Hendricks, D. W. (2006). Water treatment unit processes: Physical and chemical. CRC Press.

Hoek, G., Beelen, R., De Hoogh, K., Vienneau, D., Gulliver, J., Fischer, P., \& Briggs, D. (2008). A review of land-use regression models to assess spatial variation of outdoor air pollution. Atmospheric Environment, 42(33), 7561-7578.

Hritonenko, N., \& Yatsenko, Y. (1999). Mathematical modeling in economics, ecology and the environment. Kluwer Academic Publishers, Dordrecht, Boston, London.

Kaimal, J. C., \& Finnigan, J. J. (1994). Atmospheric boundary layer flows: Their structure and measurement. Oxford University Press.

Landau, L. D., \& Lifshitz, E. M. (2013). Course of theoretical physics. Elsevier. Miller, C. T., Dawson, C. N., Farthing, M. W., Hou, T. Y., Huang, J., Kees, C. E., \& Langtangen, H. P. (2013). Numerical simulation of water resources problems: Models, methods, and trends. Advances in Water Resources, 51, 405-437.

Prentice, I. C., Bondeau, A., Cramer, W., Harrison, S. P., Hickler, T., Lucht, W., \& Sykes, M. T. (2007). Dynamic global vegetation modeling: Quantifying terrestrial ecosystem responses to large-scale environmental change. In: Terrestrial Ecosystems in a Changing World Springer, Berlin, Heidelberg. P. 175-192.

Van Oost, K., Govers, G., \& Desmet, P. (2000). Evaluating the effects of changes in landscape structure on soil erosion by water and tillage. Landscape Ecology, 15(6), 577-589.

Boardman, W. J., \& Favis-Mortlock, D. (1998). Modelling soil erosion by water. In: Modelling Soil Erosion by Water. Springer, Berlin, Heidelberg. P. 3-6. 